\title{
Program in BASIC to combine data from two different selective detectors and its application for screening of pesticides in residue analysis
}

\author{
H.-J. Stan and H. Goebel \\ Institut für Lebensmittelchemie der Technischen Universität Berlin, Müller-Breslau-Straße, 1000 Berlin 12, FR Germany
}

\section{Introduction}

For the analysis of pesticide residues in food, gas chromatography with selective detectors is established internationally as the most suitable method. The application of electron capture (ECD) and nitrogen-phosphorus (NPD) detectors enables the selective detection of contaminants at trace level in the presence of a multitude of compounds extracted from the matrix, which do not respond to these detectors.

The number of compounds used in agriculture for plant protection and the variety of pollutants in the environment has increased to such a level that it is impossible to separate them all in a single chromatogram, despite using high-performance capillary columns. These capillary columns allow the retention times of compounds to be determined with a very high accuracy and good reproducibility. The high resolution facilitates the differentiation of substances belonging to the same structural class as organophosphate pesticides (PP) or chlorinated pesticides (CP).

When splitting the effluent of the capillary column to both selective detectors, additional information about the identity of the individual compounds can be obtained from the chromatograms by calculating the response ratios.

A concept has recently been developed for automated pesticide residue analysis - realizing it by means of a gas chromatograph with options for BASIC programming, dualchannel operation and automatic liquid sampling [1 and 2].

The BASIC program, which controls the automated analysis, calculates the residue concentrations and evaluates the degree of certainty of the pesticides found is presented here.

\section{Materials and methods}

The pesticide residue analysis was performed with a gas chromatograph (HP 5880 A, Hewlett Packard, Palo Alto, California, USA) using capillary columns (BP 1, SGE-Scientific Glass Engineering, Australia) and effluent splitting to the two selective detectors (NPD and ECD). The signals from detectors are processed in a dual-channel integrator connected to two terminals, allowing both chromatograms to be recorded in parallel. The manufacturer's operating system and chromatography programs are used for data collection, immediate recording of the chromatograms, recognition of calibrated peaks and quantitation. The internal standard method is generally used. The instrument is also equipped with an autosampler, HP $7671 \mathrm{~A}$, and the microprocessor's memory is extended by a cartridge tape unit.

The procedure of the complete pesticide analysis has already been described [1 and 2].

\section{The program}

The HP $5880 \mathrm{~A}$ was launched as the first gas chromatograph which could be programmed in BASIC; this allows it to execute individual calculations, to format reports and to control the autosampler. The authors' program was developed to fulfil the following requirements:

(1) The ability to run a food sample with parallel signal processing in two channels and storage of the data.

(2) Calculation of peaks found by means of several calibration tables.

(3) Evaluation of the results by comparing the peaks identified in the two channels.

(4) Summarizing final results in a clearly arranged report

The HP 5880 A was designed to store one calibration table in each channel. However, the large number of pesticides included in the authors' analytical method requires at least three calibration mixtures with the three corresponding calibration tables in each channel. Therefore the calibration tables have to be saved on an external memory: in this case a tape. The calibration tables are included in the three 'Analysis files'. Each file contains two calibration tables generated by dual-channel recording of one calibration mixture. Additionally, the analysis files contain the parameter settings of the instrument to run the gas chromatographic analysis. A special problem arises from the fact that communication between the two channels is limitedcalculations can easily be performed, but processing in one channel cannot be controlled by the other one. Therefore two separate programs have been created, these are synchronized by means of waiting loops.

The programs are documented in figures 1 and 2; the REM statements should make them almost self-explanatory.

For readers unfamiliar with the HP $5880 \mathrm{~A}$, a list of some of the commands for activating special procedures and functions integrated in the program might be helpful:

START AUTO SEQ X, X: Starts the automatic sampler. The two numbers define the first and last bottle.

RECALIB: The areas from the calibration mixtures found in the most recent run and the amounts from the original calibration run of the same mixture are used to calculate new response factors.

RECALIB RUN TIME: The retention times in the calibration table are replaced by real times found in the most recent run.

SAMPLE \$: Returns the number of the sampler bottle in the tray.

ID \$: Returns the sample names from the sample table. 
DETECTOR A O: NPD is switched off.

AMT (I): Returns the concentration of a specified peak calibrated.

HEAD \$: Returns the title from the calibration table.

\section{Application to a real food sample}

The automated pesticide residue analysis controlled by the BASIC program described has been designed as a screening procedure. As already mentioned, a complete residue analysis in food of unknown origin cannot be performed on a single capillary column: so the aim of automated screening is to provide the analyst with information about contaminants that may be present in the specified sample. All of the suspected pesticides listed in the final report are analysed using an independent method of confirmation [1 and 2]. Examples of final reports from the two channels are shown in figures 3 and 4 . All peaks identified by means of the data stored for each calibration mixture are printed, and those responding to both detectors are compared by applying their specific response ratios. The deviation from the value calculated from the calibration data is reported as a percentage. A difference of more than $30 \%$ is usually an indication that the peak found does not correspond to the substance calibrated. Also, all compounds identified with only one detector are indicated, as well as those belonging to 'critical pairs' in chromatography and those responding to both detectors.

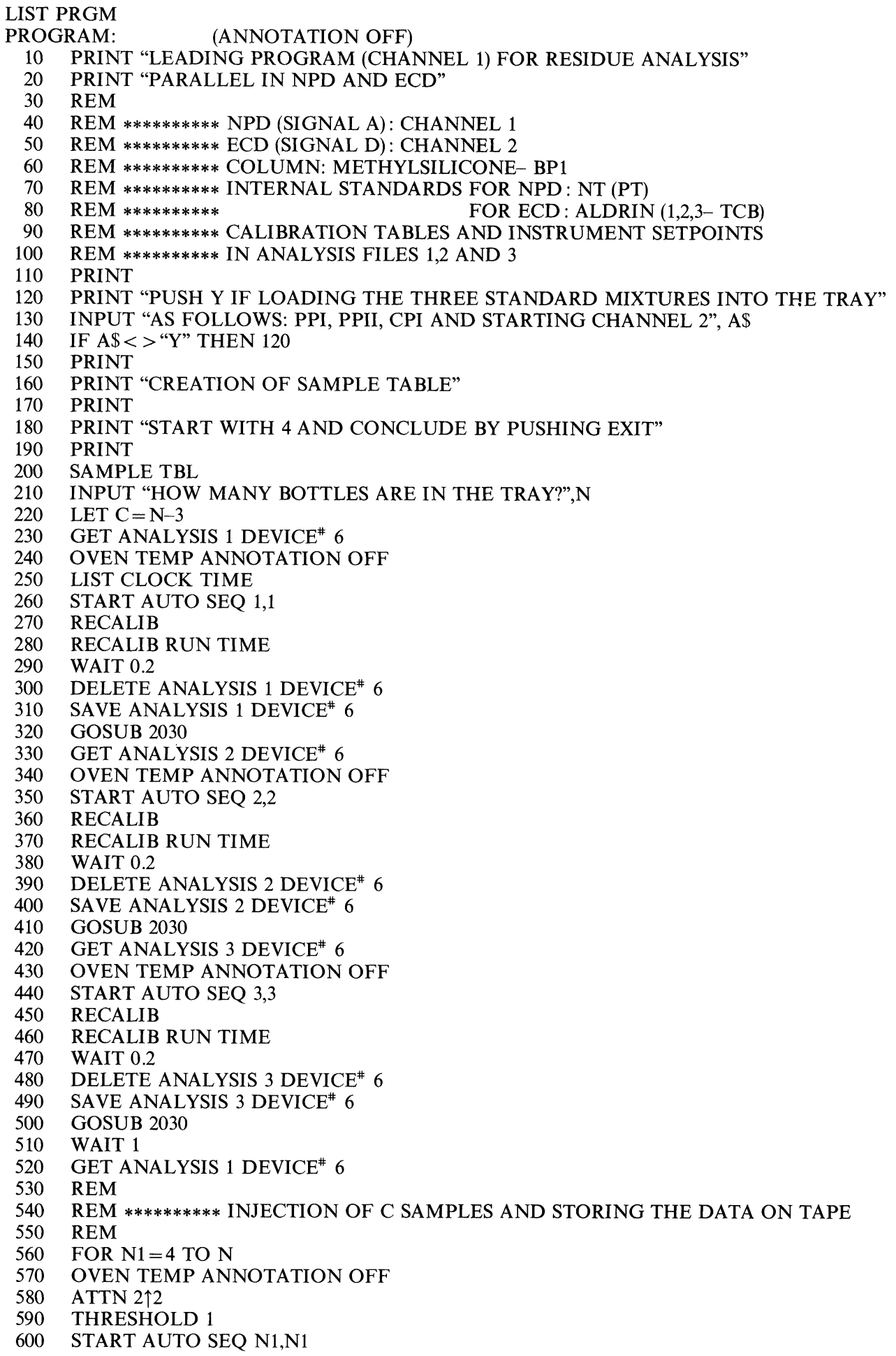




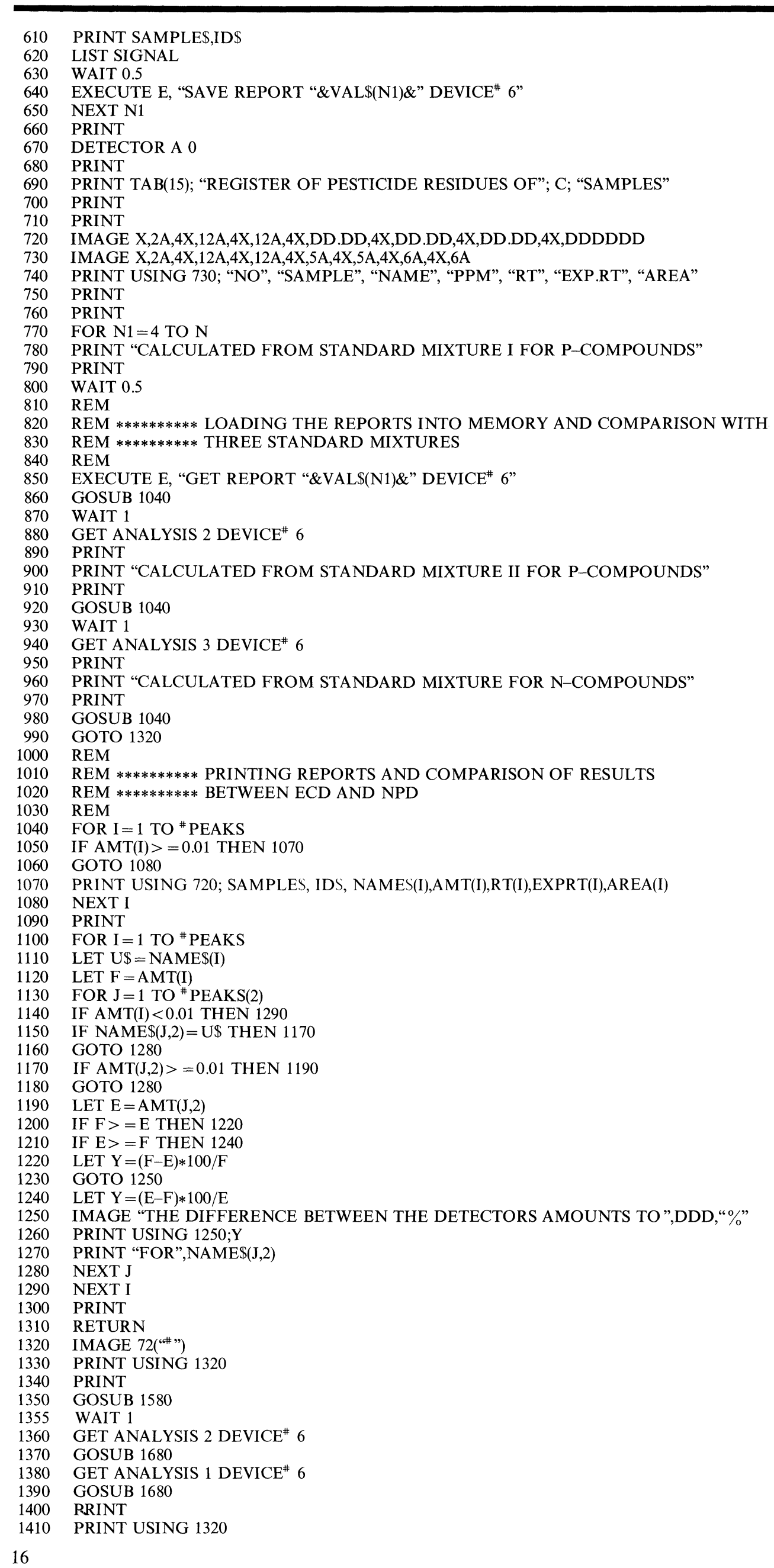




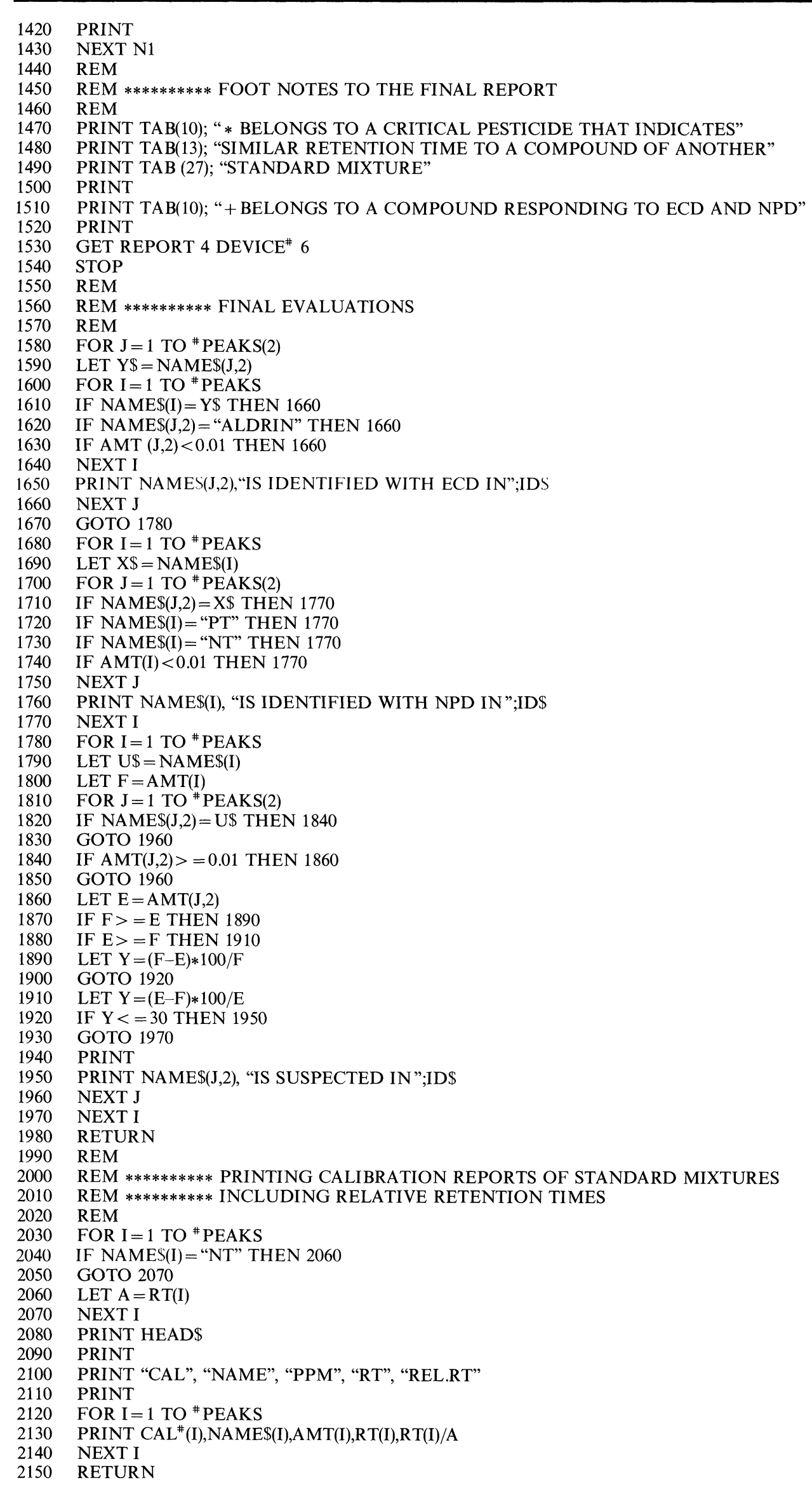

Figure 1. The program for the NPD channel (leading program). 


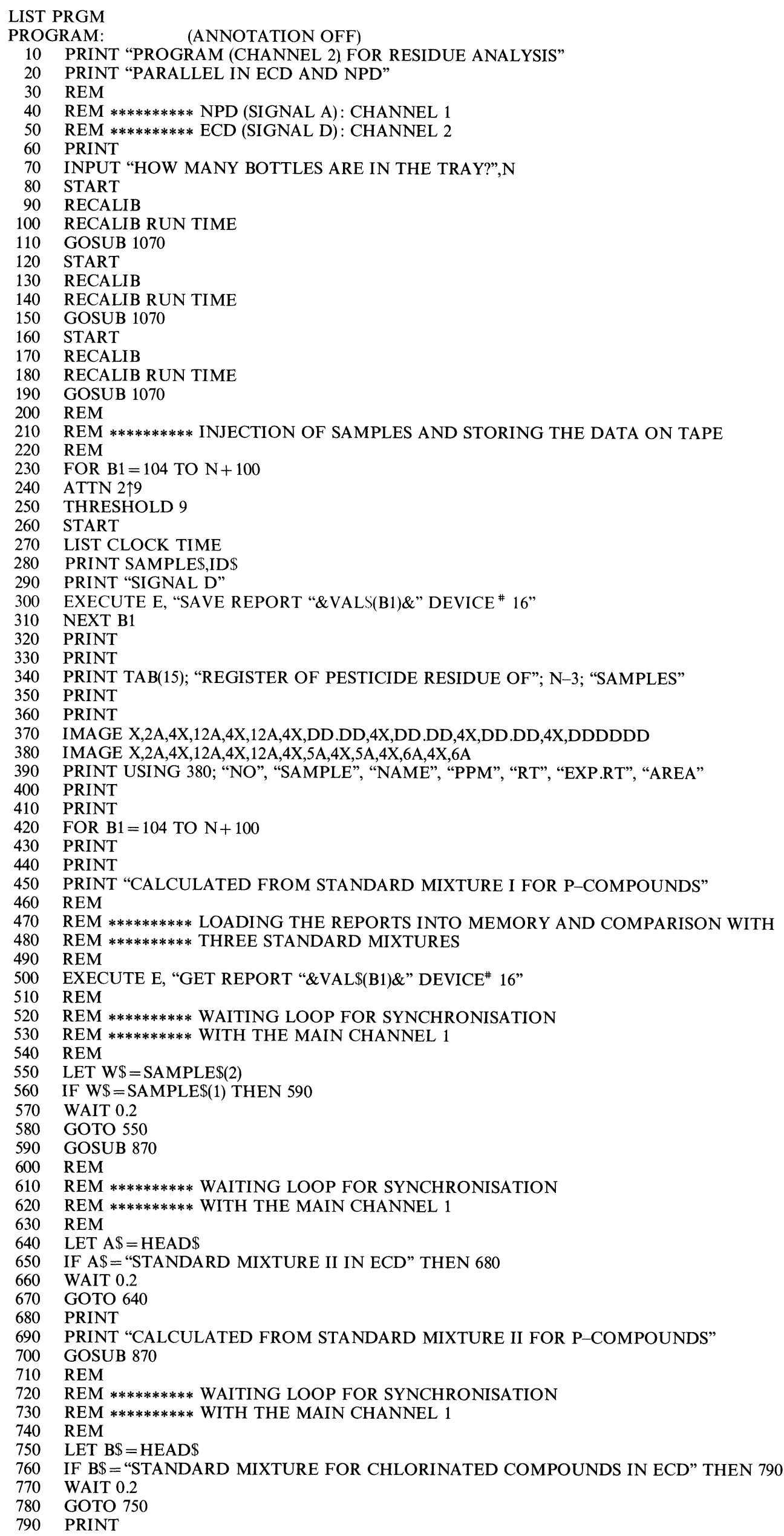




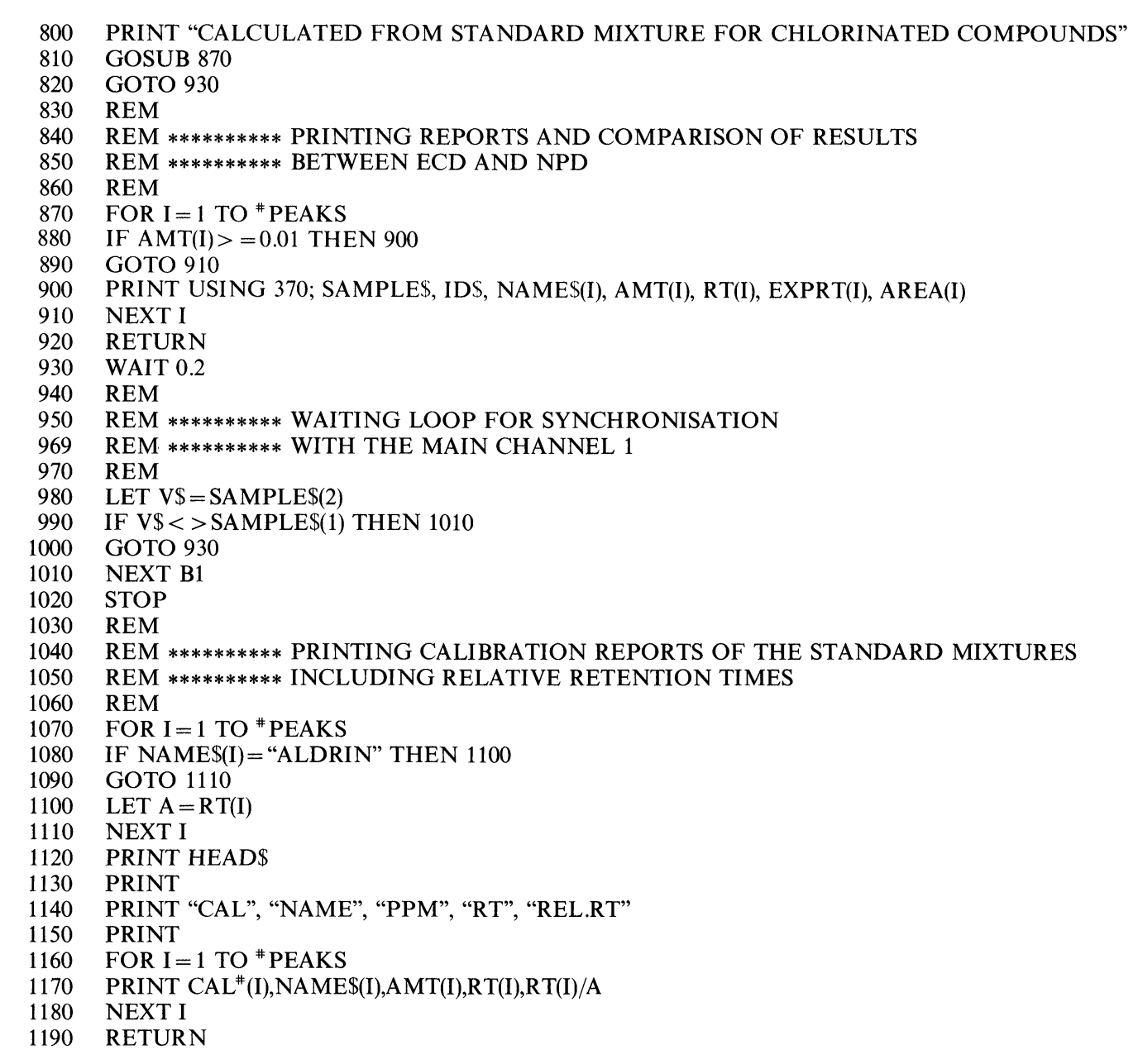

Figure 2. The program for the ECD channel.

REGISTER OF PESTICIDE RESIDUE OF 1 SAMPLES

NO SAMPLE NAME PPM RT EXP.RT AREA

CALCULATED FROM STANDARD MIXTURE I FOR P-COMPOUNDS

\begin{tabular}{|c|c|c|c|c|c|c|}
\hline 4 & PEACHES & PHOSPHAMI + & .54 & 13.97 & 13.86 & \\
\hline 4 & PEACHES & PARATH-ME +* & 1.48 & 14.21 & 14.20 & 49932 \\
\hline 4 & PEACHES & MALATHION + & 2.69 & 16.49 & 16.57 & 167 \\
\hline 4 & PEACHES & ALDRIN & 2.00 & 16.85 & 16.85 & 1009 \\
\hline 4 & PEACHES & PARATHION $+{ }^{*}$ & .20 & 17.21 & 17.14 & \\
\hline
\end{tabular}

CALCULATED FROM STANDARD MIXTURE II FOR P-COMPOUNDS

\begin{tabular}{|c|c|c|c|c|c|c|}
\hline 4 & PEACHES & DICHLOFENT $+*$ & 3.37 & 14.21 & 14.18 & 49932 \\
\hline 4 & PEACHES & PARAOXON +* & .59 & 14.98 & 15.05 & 5607 \\
\hline 4 & PEACHES & ALDRIN & 2.00 & 16.85 & 16.85 & 100997 \\
\hline 4 & PEACHES & DURSBAN $+{ }^{*}$ & .10 & 17.21 & 17.18 & 2577 \\
\hline
\end{tabular}

CALCULATED FROM STANDARD MIXTURE FOR CHLORINATED COMPOUNDS

\begin{tabular}{|c|c|c|c|c|c|c|}
\hline 4 & PEACHES & CHLORFPROP-M & .79 & 7.67 & 7.60 & 2719 \\
\hline 4 & PEACHES & HEPTACHLOR* & .30 & 14.98 & 14.97 & 5607 \\
\hline 4 & PEACHES & ALDRIN & 2.00 & 16.85 & 16.85 & 100997 \\
\hline
\end{tabular}

Figure 3. Print-out from the ECD channel for a screening run of pesticides in peaches. 


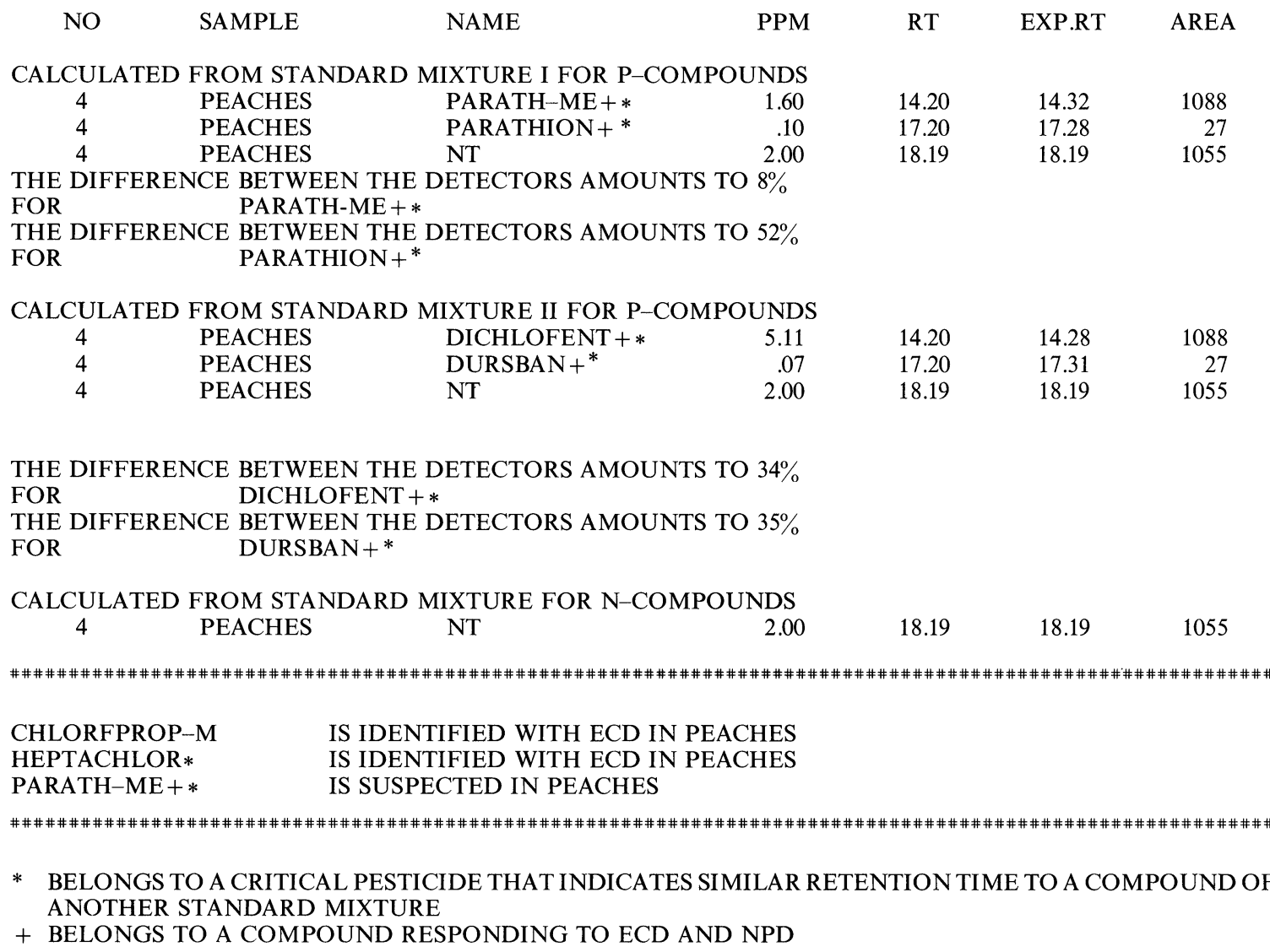

Figure 4. Print-out from the NPD channel for the same screening run as in figure 3, including final report.

\section{Discussion}

Figures 3 and 4 show the parallel print-outs from the two terminals reporting a screening run for pesticide residues in peaches. By means of this example, the utility and the limits of our program for evaluating food samples are briefly discussed.

After the clean-up, a number of substances responding to the ECD are generally found in a chromatogram and several are recognized as calibrated pesticides in the screening run. In figure 3 a total of nine pesticides are indicated, together with the internal standard (aldrin). Only these nine compounds out of about 80 pesticides incorporated in three calibration mixtures may be present in the sample. Processing of the NPD signals recorded simultaneously in the other channel results in four organophosphorous pesticides and no nitrogen-containing pesticide (figure 4). For all of the compounds responding to both detectors a calculation of the response ratios and comparison with their calibrated values leads to the discrimination of 'parathion', dichlofenthion' and 'dursban', whereas the response ratio of 'parathion methyl' is close to the calibrated one. Therefore parathion methyl is announced in the final report as suspected. This screening run permits no further decision about 'chlorfenprop methyl' and 'heptachlor'.

All pesticides marked with a cross in figure 3 are organophosphorous compounds and so they also have to respond to the NPD. As there are no corresponding signals for 'phosphamidon', 'malathion' and 'paraoxon' in the NPD report of this example, all three substances are eliminated and do not appear in the final report. This discrimination procedure results in the final proposal of chlorfenprop methyl, heptachlor and parathion methyl as possibly being present in this sample.

The program proved to be a great help in routine analysis for selecting the positive food samples after screening. A major drawback is the time-consuming data transfer between the tape and the gas chromatograph's memory.

The analyst has to inspect the chromatograms of both detectors in order to evaluate the quality of the separation and the performance of the chromatographic system. After this, the computer supports him by handling the huge amount of information produced by a series of screening runs.

\section{References}

1. Goebel, H. and Stan, H.-J. in J. Rijks (ed.), Proceedings of the Fifth International Symposium on Capillary Chromatography (Elsevier, Amsterdam, 1983), 557.

2. Stan, H.-J. and Goebel, H., Journal of Chromatography (in press). 


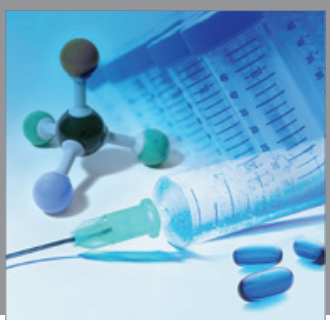

International Journal of

Medicinal Chemistry

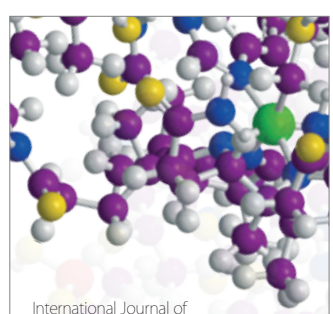

Carbohydrate Chemistry

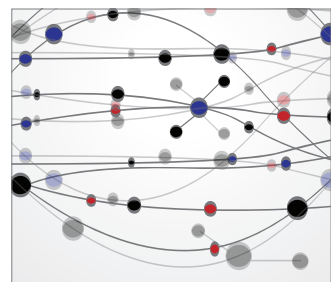

The Scientific World Journal
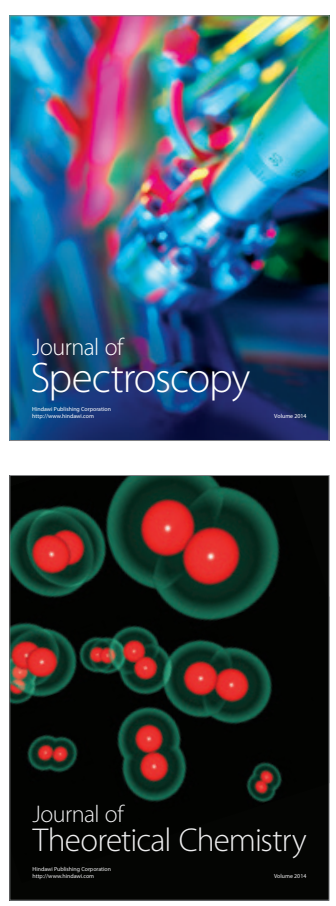
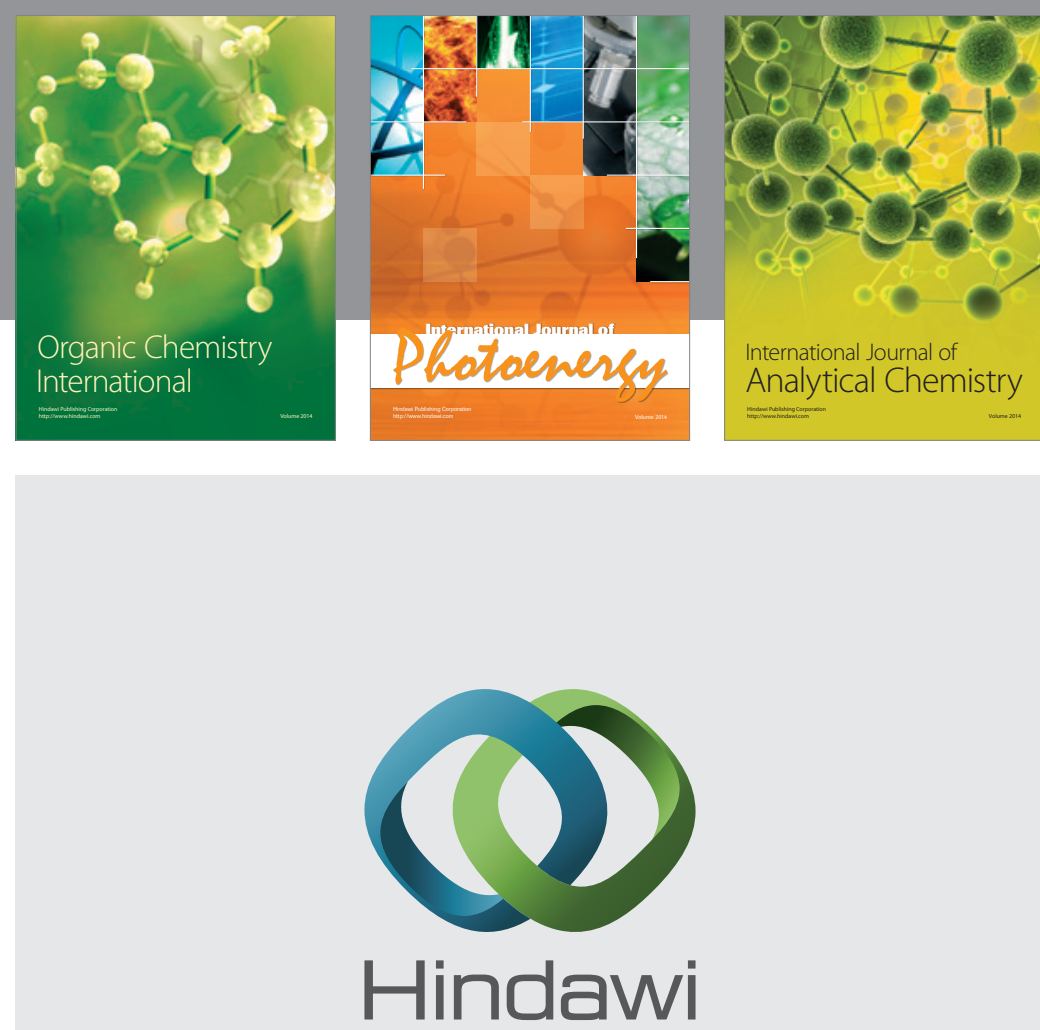

Submit your manuscripts at

http://www.hindawi.com
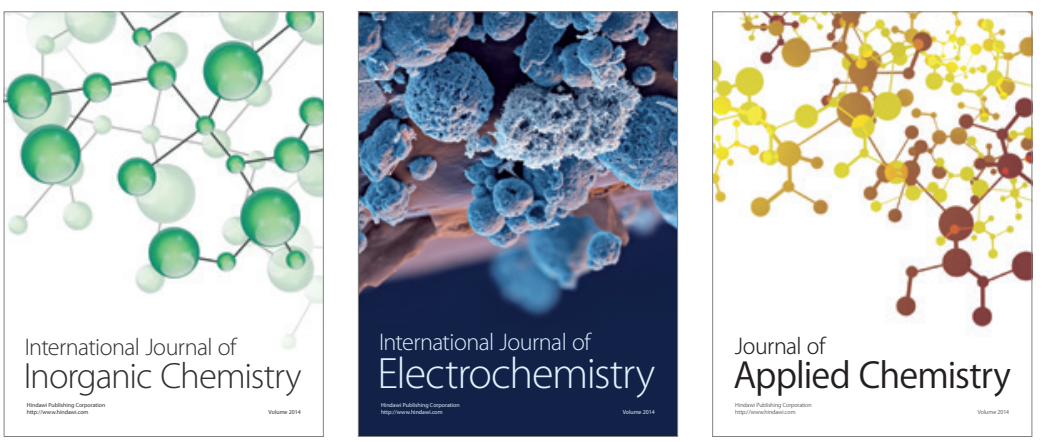

Journal of

Applied Chemistry
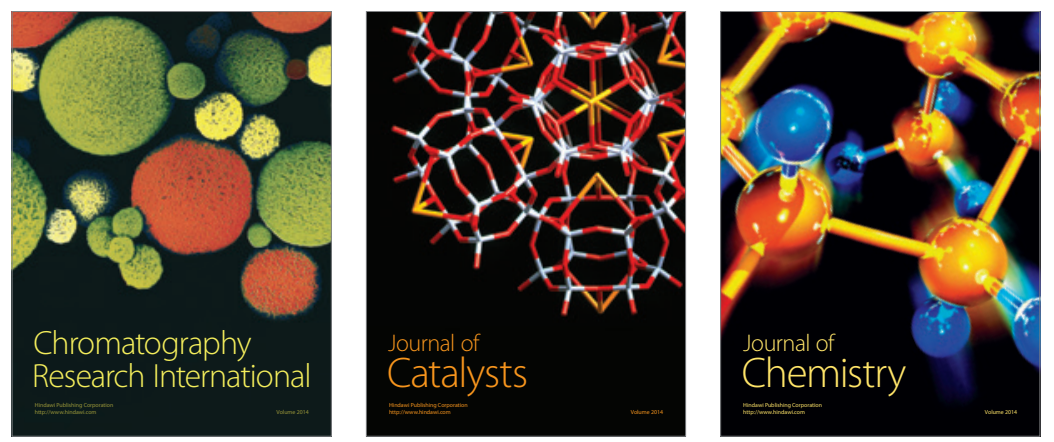
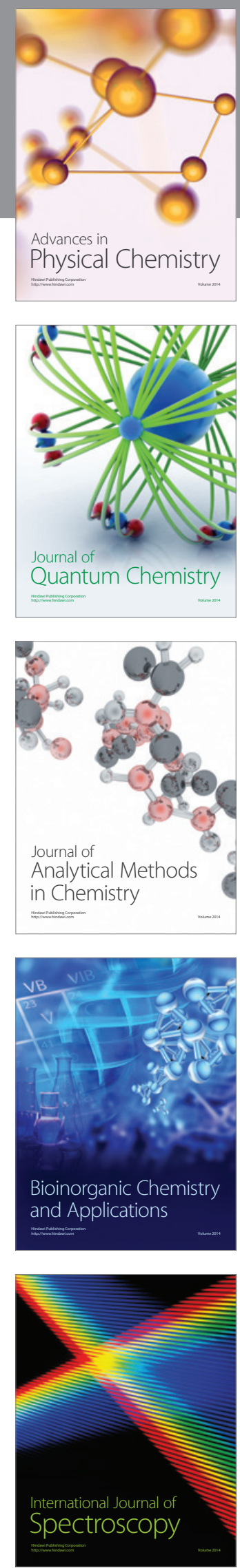